\title{
Full counting statistics of conductance for disordered systems
}

\author{
Bin Fu, ${ }^{1}$ Lei Zhang,,${ }^{1,2,3,}{ }^{*}$ Yadong Wei, ${ }^{4}$ and Jian Wang ${ }^{1, \dagger}$ \\ ${ }^{1}$ Department of Physics and the Center of Theoretical and Computational Physics, The University of Hong Kong, \\ Pokfulam Road, Hong Kong, China \\ ${ }^{2}$ State Key Laboratory of Quantum Optics and Quantum Optics Devices, Institute of Laser Spectroscopy, \\ Shanxi University, Taiyuan 030006, China \\ ${ }^{3}$ Collaborative Innovation Center of Extreme Optics, Shanxi University, Taiyuan 030006, China \\ ${ }^{4}$ College of Physics and Energy, Shenzhen University, Shenzhen 518060, China \\ (Received 9 May 2017; revised manuscript received 31 July 2017; published 7 September 2017)
}

\begin{abstract}
Quantum transport is a stochastic process in nature. As a result, the conductance is fully characterized by its average value and fluctuations, i.e., characterized by full counting statistics (FCS). Since disorders are inevitable in nanoelectronic devices, it is important to understand how FCS behaves in disordered systems. The traditional approach dealing with fluctuations or cumulants of conductance uses diagrammatic perturbation expansion of the Green's function within coherent potential approximation (CPA), which is extremely complicated especially for high order cumulants. In this paper, we develop a theoretical formalism based on nonequilibrium Green's function by directly taking the disorder average on the generating function of FCS of conductance within CPA. This is done by mapping the problem into higher dimensions so that the functional dependence of generating a function on the Green's function becomes linear and the diagrammatic perturbation expansion is not needed anymore. Our theory is very simple and allows us to calculate cumulants of conductance at any desired order efficiently. As an application of our theory, we calculate the cumulants of conductance up to fifth order for disordered systems in the presence of Anderson and binary disorders. Our numerical results of cumulants of conductance show remarkable agreement with that obtained by the brute force calculation.
\end{abstract}

DOI: 10.1103/PhysRevB.96.115410

\section{INTRODUCTION}

During the fabrication of nanoelectronic devices, it is inevitable to have randomness due to imperfections such as impurities, defects, and roughness, etc. Many quantum transport properties of nanoelectronic devices, such as electric current, conductance, and electrochemical capacitance, are strongly influenced by the disorder effects [1-4]. In addition, nanoelectronic device performance depends very much on the device-to-device variability which can be very large due to the disorder effects [5-8]. Thus the study of disorder effects in quantum transport is extremely important in understanding and controlling the reliability of nanoelectronic devices. On the other hand, understanding disorder effects is also a fundamental issue in condensed matter physics [9-13]. For instance, one of the hallmarks in mesoscopic physics is the universal conductance fluctuation in the diffusive regime in the presence of disorders [14-19]. Due to the quantum interference, the sample to sample fluctuation of order $e^{2} / h$ is a universal value which depends only on the dimensionality and the symmetry of the system [20].

It is known that in mesoscopic systems or nanoscale systems quantum effects dominate. Since the quantum transport is a stochastic process in nature, the current can fluctuate even in the clean mesoscopic system giving rise to shot noise at zero temperature. In principle, the random process can be fully understood by studying the corresponding distribution function [21]. If the distribution function of the physical quantity is Gaussian, the average value and second cumulant are enough to

\footnotetext{
*zhanglei@sxu.edu.cn

†jianwang@hku.hk
}

describe the distribution. However, the distribution of electric current (or conductance) is usually not Gaussian. Hence in general, all cumulants of current (called full counting statistics, or FCS) have to be included in order to fully characterize the electronic quantum transport process.

When both disorders and quantum effects are present in the system, disorder average as well as quantum average are needed giving rise to a hierarchy of interesting quantities. For instance, for the clean sample, we have conductance, shot noise, third order cumulant, etc. In particular, the shot noise is the second order cumulant due to the quantum fluctuation [22]. For disorder samples, we can study disorder averaged conductance, disordered average of shot noise, etc. Clearly the next level in the hierarchy is the conductance fluctuation which is the second cumulant due to the disorder fluctuation, etc.

In order to perform disorder average on various transport quantities, a large number of configurations are needed to obtain accurate estimate. This is the so-called brute force calculation which is very time consuming for large system size. For nanoelectronic devices, the doping concentration $p$ is usually very small. If $N_{0}$ is the total number of lattice site in the system and $p=1 / N_{0}$, then there is only one impurity in the system. This would make the brute force calculation impossible to perform if $p<1 / N_{0}$ since the number of impurities in the system is less than one. Due to these reasons, approximated methods were used to perform analytic disorder average. The coherent potential approximation (CPA) is one of the successful examples which calculates the average of single Green's function [23-26].

To calculate transport properties such as average conductance, the method of diagrammatic perturbation expansion was used. It turns out that to get disorder averaged $n$th order 
cumulant of conductance using diagrammatic perturbation expansion, one has to calculate disorder averaged $2 n$ Green's function correlator. For example, since the functional dependence of conductance on the Green's function is quadratic, one needs to calculate disorder averaged of two Green's function correlators to obtain the averaged conductance. For a similar reason, four Green's function correlators are needed for the shot noise [7,27]. Various techniques have been developed to calculate the $2 n$ Green's function correlator within CPA. For instance, using the nonequilibrium vertex correction (NVC) within CPA [28-31], the averaged conductance and transient current [32] involving two Green's function correlators were studied. A theory of generalized nonequilibrium vertex correction (GNVC) within CPA was developed to study the disorder averaged shot noise and transmission fluctuation [27,33]. Recently, disorder averaged third and fourth order moments have also been investigated in Ref. [34] using diagrammatic expansion. Since the method of diagrammatic expansion is extremely complicated, it is clearly not practical to move along this direction for studying high order cumulants in the presence of disorders.

The full-counting statistics (FCS) is an elegant way to study the current correlations and yields not only the shot noise but all high order cumulants [35-37]. The key quantity in FCS is the generating function, from which all cumulants can be obtained. In this paper, we have developed a theoretical formalism to calculate disorder averaged generating function within CPA without using complicated diagrammatic technique. This is done through dimension expansion which maps the nonlinear functional dependence of generating function on Green's function into the linear dependence in higher dimensions. By doing this, the diagrammatic perturbation method is completed avoided since the average of a single Green's function is done through CPA. This formalism allows us to calculate the averaged cumulant to any desired order efficiently. As an illustration, we implement our theoretical formalism in the tight binding model to calculate the disorder averaged high order cumulants by considering Anderson and binary type disorders. We have shown theoretically that for the average conductance our formalism gives the same result as that of CPA-NVC [28-31]. Our numerical results show that the transmission fluctuation obtained from our theory is the same as that from the GNVC method [27,33]. Finally for disorder averaged high order cumulants, our results are very accurate compared with the brute force calculation.

The paper is organized as follows. In Sec. II, we introduce the theoretical formalism for calculating the generating function of FCS and hence high order cumulant of conductance of a disordered system can be obtained. As an example, we apply our method to the tight binding model on a square lattice and show the numerical results in Sec. III. Finally, Sec. IV is our discussion and conclusion part.

\section{THEORETICAL FORMALISM}

\section{A. Coherent potential approximation}

Before presenting our theory of FCS in the presence of disorders, we will first briefly review the coherent potential approximation (CPA) approach [23-27,38]. Suppose the

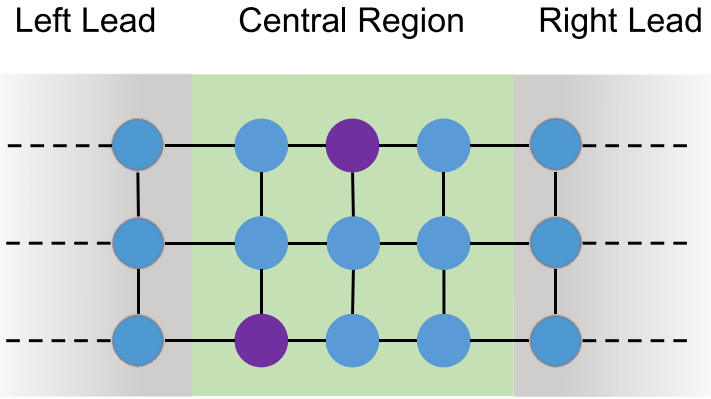

FIG. 1. Schematic plot of a two terminal system having some impurities (purple sites) in the central region. The left and right leads extend to infinity.

Hamiltonian of the disordered system shown schematically in Fig. 1 is given by

$$
H=H_{0}+v+H_{\text {lead }}+H_{T},
$$

where $H_{0}$ is the unperturbed Hamiltonian of the central region and $v=\sum_{i}|i\rangle v^{i i}\langle i|$ describes the on-site random disorder and $i$ denotes the specific site in the central region, $H_{\text {lead }}$ is the Hamiltonian of the leads, and $H_{T}$ is the coupling between the central region and leads.

The key idea of the CPA method is to replace the disorder averaged Green's function $\left\langle G^{r}\right\rangle$ by a renormalized Green's function $G_{e}^{r}$ where the on-site potential matrix is shifted by $\Delta E$ which is a diagonal matrix [39],

$$
\begin{aligned}
\left\langle G^{r}\right\rangle=G_{e}^{r} & \equiv \frac{1}{(E-\Delta E)-H_{0}-\Sigma^{r}} \\
& =\frac{1}{\left(G_{0}^{r}\right)^{-1}-\Delta E},
\end{aligned}
$$

where \langle\rangle stands for disorder configuration average; $\Sigma^{r}$ is the self energy of the leads; $G_{0}^{r}=\left[G_{0}^{a}\right]^{\dagger}=\left(E-H_{0}-\Sigma^{r}\right)^{-1}$ is the retarded Green function in the absence of disorders.

Instead of performing the time consuming brute force calculation of $\left\langle G^{r}\right\rangle$, we wish to compute the renormalized quantity $\Delta E$ from Eq. (2). Notice that the Hamiltonian of the disordered system can be rewritten as

$$
H=\left(H_{0}+H_{\text {lead }}+H_{T}+\Delta E\right)+(v-\Delta E) .
$$

By doing so, it is easy to obtain a Dyson equation for $G^{r}$ [40],

$$
G^{r}=G_{e}^{r}+G_{e}^{r} T^{r} G_{e}^{r},
$$

where $T^{r}$ is

$$
\begin{aligned}
T^{r} & =(v-\Delta E)+(v-\Delta E) G_{e}^{r}(v-\Delta E)+\cdots \\
& =(v-\Delta E)\left(I+G_{e}^{r} T^{r}\right) .
\end{aligned}
$$

Taking the configuration average on Eq. (4), we have

$$
\left\langle G^{r}\right\rangle=G_{e}^{r}+G_{e}^{r}\left\langle T^{r}\right\rangle G_{e}^{r} .
$$

Since $\left\langle G^{r}\right\rangle=G_{e}^{r}$ as defined in Eq. (2), we arrive at

$$
\left\langle T^{r}\right\rangle=0,
$$


which is regarded as the CPA condition. By taking the average of Eq. (5), $\left\langle T^{r}\right\rangle$ can be rewritten as [27]

$$
\begin{aligned}
\left\langle T^{r}\right\rangle= & \sum_{i}\left\langle t^{r, i i}\right\rangle+\sum_{i} \sum_{j \neq i}\left\langle t^{r, i i} G_{e}^{r} t^{r, j j}\right\rangle \\
& +\sum_{i} \sum_{j \neq i} \sum_{k \neq j}\left\langle t^{r, i i} G_{e}^{r} t^{r, j j} G_{e}^{r} t^{r, k k}\right\rangle \\
& +\sum_{i} \sum_{j \neq i} \sum_{k \neq j} \sum_{l \neq k}\left\langle t^{r, i i} G_{e}^{r} t^{r, j j} G_{e}^{r} t^{r, k k} G_{e}^{r} t^{r, l l}\right\rangle+\cdots
\end{aligned}
$$

where $t^{r, i i}$ is defined as

$$
\begin{aligned}
t^{r, i i} & =\left(v^{i i}-\Delta E^{i i}\right) Q^{r, i i}, \\
Q^{r, i i} & =\left[I-G_{e}^{r, i i}\left(v^{i i}-\Delta E^{i i}\right)\right]^{-1} .
\end{aligned}
$$

Physically, scattering events between different sites are coupled together as described by the second and high order terms in Eq. (8). In general Eq. (7) and Eq. (8) can be used to calculate $\Delta E$. However, there are many quantities to calculate such as $\left\langle t^{r, i i}\right\rangle$, the second order term $\left\langle t^{r, i i} G_{e}^{r} t^{r, j j}\right\rangle$ as well as higher order terms. In order to decouple them and obtain a closed form equation for $\Delta E$, people usually use the so-called single-site approximation (SSA). By taking SSA, Eq. (8) becomes

$$
\left\langle T^{r}\right\rangle=\sum_{i}\left\langle t^{r, i i}\right\rangle+\sum_{i} \sum_{j \neq i}\left\langle t^{r, i i}\right\rangle G_{e}^{r}\left\langle t^{r, j j}\right\rangle+\cdots
$$

Note that the first nonzero correlation in Eq. (8) comes from the fourth order term $(i=k, j=l)$. Thus the SSA is quite accurate. Physically, the error occurs when multiple scattering from the same site becomes important [40].

Therefore, it is easy to see that $\left\langle T^{r}\right\rangle=0$ leads to $\left\langle t^{r, i i}\right\rangle=$ 0 from SSA and CPA conditions. Finally, one can get the renormalized on-site potential [40]

$$
\begin{aligned}
\Delta E^{i i} & =\left\langle v^{i i} Q^{r, i i}\right\rangle \\
& =\int d v^{i i} \rho\left(v^{i i}\right) v^{i i} Q^{r, i i},
\end{aligned}
$$

where $\rho\left(v^{i i}\right)$ is the distribution function of disorders. Note that Eq. (11) should be solved self-consistently since $Q^{r, i i}$ contains $\Delta E^{i i}$. Once we obtain the renormalized on-site potential $\Delta E^{i i}$, the configurational average of retarded (advanced) Green functions and other physical quantities related to retarded (advanced) Green's functions could be calculated.

\section{B. Full counting statistics of disordered system}

The most important quantity in FCS is the generating function, from which various cumulants can be calculated. The steady state current generating function $Z_{V}$ can be expressed as [41]

$$
\begin{aligned}
\ln Z_{V}(\lambda)= & \int \frac{d E}{2 \pi} \operatorname{Tr} \ln \left\{I+\hat{T}(E)\left[\left(e^{i \lambda}-1\right)\left(1-f_{R}(E)\right) f_{L}(E)\right.\right. \\
& \left.\left.+\left(e^{-i \lambda}-1\right)\left(1-f_{L}(E)\right) f_{R}(E)\right]\right\},
\end{aligned}
$$

where $\hat{T}(E)=G^{r} \Gamma_{R} G^{a} \Gamma_{L}$ is the transmission matrix; $\ln Z_{V}$ is the cumulant generating function (CGF) for the current that depends on the applied voltage; $\Gamma_{\alpha}$ and $f_{\alpha}(E)$ are line width function and the Fermi distribution function of the $\alpha$ th lead, respectively.

By expanding the Fermi function in terms of bias voltage at zero temperature, one can easily find the CGF for conductance (or transmission coefficient),

$$
\ln Z(\lambda)=\operatorname{Tr} \ln \left[I+\hat{T}(E)\left(e^{i \lambda}-1\right)\right] .
$$

This CGF can be used to calculate the transmission coefficient and its corresponding high order cumulants for clean samples by taking derivatives of $\ln Z(\lambda)$ with respect to $i \lambda$.

Our aim is to calculate the disorder averaged high order cumulant of transmission coefficient using the FCS method. For this purpose, we rewrite the CGF of Eq. (13) as

$$
\ln Z(\lambda)=\ln \operatorname{Det}(I+G A),
$$

where $A$ and Green's function $G$ are defined as

$$
\begin{gathered}
A=A_{0} \zeta, \\
A_{0}=\left(\begin{array}{cc}
0 & -\Gamma_{R} \\
\Gamma_{L} & 0
\end{array}\right), \\
G=\left(\begin{array}{cc}
G^{r} & 0 \\
0 & G^{a}
\end{array}\right),
\end{gathered}
$$

with $\zeta=\sqrt{\left(e^{i \lambda}-1\right)}$. It is easy to see that Eqs. (14) and (13) are the same. We see from Eq. (14) that the functional dependence of cumulant generating function on Green's function is nonlinear. However, as will be clear later, defining Green's function $G$ in higher dimensions is crucial to make the linear functional dependence of CGF on the Green's function [see Eq. (20)].

It is easy to verify that the derivatives of $\ln Z$ in Eq. (14) correspond to different orders of cumulant $C_{n}=\left.\frac{\partial^{n} \ln Z}{\partial(i \lambda)^{n}}\right|_{\lambda=0}$. For example, the first three order cumulants are

$$
\begin{aligned}
& C_{1}=T=\operatorname{Tr}(\hat{T}), \\
& C_{2}=\operatorname{Tr}\left(\hat{T}-\hat{T}^{2}\right), \\
& C_{3}=\operatorname{Tr}\left(2 \hat{T}^{3}-3 \hat{T}^{2}+\hat{T}\right) .
\end{aligned}
$$

Here the first order cumulant is just a transmission coefficient which contains two Green's functions whose random average can be done using CPA-NVC, a diagrammatic perturbation expansion method. The second order cumulant is the shot noise and the third order cumulant is the skewness which provides the information on asymmetry of the transmission distribution about its mean value.

In the following, we will show that the generating function $\ln Z(\lambda)$ in Eq. (14) is easily coupled with the CPA method to calculate disorder averaged transmission coefficient and its high order cumulants. Note that the $\mathrm{CGF} \ln Z(\lambda)$ can be expressed as

$$
\ln Z(\lambda)=\operatorname{Tr} \ln (I+G A)=\operatorname{Tr}\left(\int_{0}^{1} \frac{d x}{G^{-1}+A x} A\right) .
$$

Introducing a generalized Green's function $\mathcal{G}(x)=$ $\left(G^{-1}+A x\right)^{-1}$, the CGF finally becomes

$$
\ln Z(\lambda)=\operatorname{Tr}\left(\int_{0}^{1} d x \mathcal{G}(x) A\right)
$$


Note that $\mathcal{G}(x)$ is neither retarded nor advanced. It is designed as the ideal form to perform the disorder average using CPA. From Eq. (20) we see that we have mapped the original problem of nonlinear functional dependence of CGF on Green's function into a linear dependent problem in high dimensional space, allowing us to facilitate the random average just using CPA. Clearly the configuration averaged cumulants can be obtained from

$$
\left\langle C_{n}\right\rangle=\left.\frac{\partial^{n}\langle\ln Z\rangle}{\partial(i \lambda)^{n}}\right|_{\lambda=0} .
$$

Since $A$ is independent of disorder configuration, the averaged $C G F\langle\ln Z\rangle$ is expressed as

$$
\langle\ln Z\rangle=\operatorname{Tr}\left(\int_{0}^{1} d x\langle\mathcal{G}(x)\rangle A\right),
$$

where the averaged Green's function $\langle\mathcal{G}(x)\rangle$ can be calculated through CPA approach discussed in the last section. We have

$$
\langle\mathcal{G}(x)\rangle=\left(G_{0}^{-1}+A x-\Delta \mathcal{E}\right)^{-1},
$$

where $G_{0}$ and $\Delta \mathcal{E}$ are defined as

$$
\begin{gathered}
G_{0}=\left(\begin{array}{cc}
G_{0}^{r} & 0 \\
0 & G_{0}^{a}
\end{array}\right), \\
\Delta \mathcal{E}=\left(\begin{array}{cc}
\Delta \mathcal{E}_{11} & \Delta \mathcal{E}_{12} \\
\Delta \mathcal{E}_{21} & \Delta \mathcal{E}_{22}
\end{array}\right) .
\end{gathered}
$$

Here $\Delta \mathcal{E}_{\alpha \beta}$ is the sub-block matrix of $\Delta \mathcal{E}$ which is diagonal. Comparing with the traditional renormalized on-site potential $\Delta E^{i i}$, the on-site potential for CGF $\Delta \mathcal{E}^{i i}$ is given by

$$
\Delta \mathcal{E}^{i i}=\left(\begin{array}{cc}
\Delta \mathcal{E}_{11}^{i i} & \Delta \mathcal{E}_{12}^{i i} \\
\Delta \mathcal{E}_{21}^{i i} & \Delta \mathcal{E}_{22}^{i i}
\end{array}\right)
$$

Similar to Eq. (11), we find that the renormalized on-site potential $\Delta \mathcal{E}$ satisfies the following equation

$$
\Delta \mathcal{E}^{i i}=\int d v^{i i} \rho\left(v^{i i}\right) v^{i i}\left[I-\langle\mathcal{G}(x)\rangle^{i i}\left(v^{i i} I-\Delta \mathcal{E}^{i i}\right)\right]^{-1} \text {. }
$$

To summarize, we first solve $\Delta \mathcal{E}$ from Eq. (27) which depends on $\zeta$ (or $\lambda$ ) through matrix $A$ defined in Eq. (15). Secondly, the averaged CGF can be obtained by calculating $\langle\mathcal{G}(x)\rangle$ from Eqs. (22) and (23). Finally, we calculate the disorder averaged cumulants $\left\langle C_{n}\right\rangle$ to any desired order by taking the derivative of CGF with respect to $i \lambda$.

From Eqs. (23) and (27), we see that $\Delta \mathcal{E}$ depends only on $\zeta x$ instead of $\zeta$ (or $\lambda$ ) and $x$ separately, i.e.,

$$
\Delta \mathcal{E}=\Delta \mathcal{E}(\zeta x)
$$

Similarly we have $\mathcal{G}=\mathcal{G}(\zeta x)$ as well. Using this fact, we can further re-express Eq. (22) as

$$
\langle\ln Z(\lambda)\rangle=\operatorname{Tr}\left(\int_{0}^{\zeta(\lambda)} d x\langle\mathcal{G}(x)\rangle A_{0}\right) .
$$

Thus the disorder averaged cumulants $\left\langle C_{n}\right\rangle$ can be calculated by taking $(n-1)$ th order numerical derivative of

$$
\frac{\partial\langle\ln Z\rangle}{\partial i \lambda}=\frac{1}{2} \frac{e^{i \lambda}}{\sqrt{e^{i \lambda}-1}} \operatorname{Tr}\left(\langle\mathcal{G}(\zeta)\rangle A_{0}\right)
$$

with respective to $i \lambda$ and then set $\lambda$ to zero. Hence the integral of $x$ in Eq. (22) is avoided during the numerical calculation. The advantage of using Eq. (30) to study FCS in disordered systems is that the calculation is straightforward and easy to implement. However, to calculate the cumulants $\left\langle C_{n}\right\rangle$, one needs to perform numerical derivative using finite difference method. Our results show that we have to converge the calculation of $\Delta \mathcal{E}$ to a very high precision in order to obtain accurate value of cumulants $\left\langle C_{n}\right\rangle$ for large $n$. For instance, in order to calculate the fifth order cumulant with three significant figures we have to converge $\Delta \mathcal{E}$ at precision $10^{-10}$ and it costs several tens of steps to converge at this precision. This can be time consuming. In the subsection $\mathrm{D}$ below, we will use a straightforward expansion method to avoid taking numerical derivatives which makes the calculation much more efficient.

In general, the above theoretical formalism can be easily implemented and applied to study the Anderson disorder or binary type of disorders. The numerical results calculated using this formalism, termed as FCS-CPA, will be presented in Sec. III. Numerically, the first order cumulant shows excellent agreement with the results obtained using traditional CPA plus nonequilibrium vertex correction (NVC) method [7] while our second order cumulant is numerically the same as that obtained from the GNVC method [27]. In fact, we can show analytically that the FCS-CPA method up to the first order in the counting field $\lambda$ is the same as that of the CPA-NVC method.

\section{Example: First order cumulant}

In this subsection, we will illustrate how to calculate the first cumulant of FCS-CPA and therefore establish equivalence between our method and the CPA-NVC. Since we will calculate the first order cumulant, it is natural to expand $\langle\mathcal{G}(x)\rangle$ in terms of $\lambda$. First, we have $e^{i \lambda}-1 \approx i \lambda$ in Eq. (15) and define a new parameter $\delta=\sqrt{i \lambda}$. The renormalized on-site potential can be expressed as

$$
\Delta \mathcal{E}=\Delta \mathcal{E}_{0}+\Delta \mathcal{E}_{1} x \delta,
$$

where the subscript of $\Delta \mathcal{E}$ denotes the expansion order of $\delta$. Up to this order, the averaged Green's function $\langle\mathcal{G}\rangle$ [Eq. (23)]) is written as

$$
\langle\mathcal{G}(x)\rangle=\left(\mathcal{G}_{e}^{-1}+\left(A_{0}-\Delta \mathcal{E}_{1}\right) x \delta\right)^{-1},
$$

where we have introduced the generalized CPA Green's function $\mathcal{G}_{e}^{-1}=G_{0}^{-1}-\Delta \mathcal{E}_{0}$. Expanding Eq. (32) up to the first order in $\delta$, we arrive at

$$
\langle\mathcal{G}(x)\rangle=\mathcal{G}_{e}-\mathcal{G}_{e}\left(A_{0}-\Delta \mathcal{E}_{1}\right) \mathcal{G}_{e} x \delta .
$$

From the CPA equation in Eq. (27), we have

$$
\left\langle\left(v^{i i} I-\Delta \mathcal{E}^{i i}\right)\left[I-\langle\mathcal{G}(x)\rangle^{i i}\left(v^{i i} I-\Delta \mathcal{E}^{i i}\right)\right]^{-1}\right\rangle=0 .
$$

The explicit equations determining $\Delta \mathcal{E}_{0}$ and $\Delta \mathcal{E}_{1}$ can be obtained as follows.

Plugging Eqs. (31) and (33) into Eq. (34), we find

$$
\begin{aligned}
0= & \left\langle\mathcal{T}^{i i}\right\rangle-\Delta \mathcal{E}_{1}^{i i}\left\langle\mathcal{Q}^{i i}\right\rangle x \delta-\left\langle\mathcal{T}^{i i} \mathcal{G}_{e}^{i i} \Delta \mathcal{E}_{1}^{i i} \mathcal{Q}^{i i}\right\rangle x \delta \\
& -\left\langle\mathcal{T}^{i i}\left[\mathcal{G}_{e}\left(A_{0}-\Delta \mathcal{E}_{1}\right) \mathcal{G}_{e}\right]^{i i} \mathcal{T}^{i i}\right\rangle x \delta,
\end{aligned}
$$

where we have defined

$$
\mathcal{T}^{i i}=\left(v^{i i} I-\Delta \mathcal{E}_{0}^{i i}\right) \mathcal{Q}^{i i},
$$


and

$$
\mathcal{Q}^{i i}=\left[I-\mathcal{G}_{e}^{i i}\left(v^{i i} I-\Delta \mathcal{E}_{0}^{i i}\right)\right]^{-1} .
$$

Note that the following relation holds

$$
\mathcal{Q}^{i i}-\mathcal{G}_{e}^{i i} \mathcal{T}^{i i}=I \text {. }
$$

Setting the zeroth order term of $x \delta$ in Eq. (35) to zero, i.e., $\left\langle\mathcal{T}^{i i}\right\rangle=0$, we have

$$
\Delta \mathcal{E}_{0}^{i i}=\left\langle v^{i i} \mathcal{Q}^{i i}\right\rangle,
$$

where we have used the following relation $[38,40]$

$$
\left\langle\mathcal{Q}^{i i}\right\rangle=I .
$$

Note that Eq. (39) is exactly the CPA equation for $\mathcal{G}_{e}$ similar to Eq. (11). Since the Green's function $G_{0}$ is the block diagonal matrix and we only consider on-site disorders, both $\Delta \mathcal{E}_{0}^{i i}$ and $\mathcal{Q}^{i i}$ should be two by two diagonal matrices. This shows that Eq. (39) can be further expressed by the following two equations

$$
\begin{aligned}
\Delta \mathcal{E}_{0,11}^{i i} & =\left\langle v^{i i}\left[I-G_{e}^{r, i i}\left(v^{i i} I-\Delta \mathcal{E}_{0,11}^{i i}\right)\right]^{-1}\right\rangle, \\
\Delta \mathcal{E}_{0,22}^{i i} & =\left\langle v^{i i}\left[I-G_{e}^{a, i i}\left(v^{i i} I-\Delta \mathcal{E}_{0,22}^{i i}\right)\right]^{-1}\right\rangle .
\end{aligned}
$$

From Eq. (41), we immediately see that $\Delta \mathcal{E}_{0,11}^{i i}$ is exactly $\Delta E^{i i}$, i.e., the solution of the traditional CPA equation [Eq. (11)]. Furthermore $\Delta \mathcal{E}_{0,22}^{i i}$ is its complex conjugate. As a result, it is enough to solve the traditional CPA and then construct the two by two matrix $\Delta \mathcal{E}_{0}^{i i}$.

For the first order term of Eq. (35) of $x \delta$, we have

$$
-\Delta \mathcal{E}_{1}^{i i}=\left\langle\mathcal{T}^{i i} \mathcal{G}_{e}^{i i} \Delta \mathcal{E}_{1}^{i i} \mathcal{Q}^{i i}\right\rangle+\left\langle\mathcal{T}^{i i}\left[\mathcal{G}_{e}\left(A_{0}-\Delta \mathcal{E}_{1}\right) \mathcal{G}_{e}\right]^{i i} \mathcal{T}^{i i}\right\rangle \text {. }
$$

We can further expand the second term on the right hand side of Eq. (42) to have

$$
\begin{aligned}
-\Delta \mathcal{E}_{1}^{i i}= & \left\langle\mathcal{T}^{i i}\left[\mathcal{G}_{e} A_{0} \mathcal{G}_{e}\right]^{i i} \mathcal{T}^{i i}\right\rangle-\sum_{k \neq i}\left\langle\mathcal{T}^{i i} \mathcal{G}_{e}^{i k} \Delta \mathcal{E}_{1}^{k k} \mathcal{G}_{e}^{k i} \mathcal{T}^{i i}\right\rangle \\
& -\left\langle\mathcal{T}^{i i} \mathcal{G}_{e}^{i i} \Delta \mathcal{E}_{1}^{i i} \mathcal{G}_{e}^{i i} \mathcal{T}^{i i}\right\rangle+\left\langle\mathcal{T}^{i i} \mathcal{G}_{e}^{i i} \Delta \mathcal{E}_{1}^{i i} \mathcal{Q}^{i i}\right\rangle
\end{aligned}
$$

Using Eq. (38) and $\left\langle\mathcal{T}^{i i}\right\rangle=0$, the last two terms of Eq. (43) cancel each other and we finally arrive at

$$
\Delta \mathcal{E}_{1}^{i i}=-\left\langle\mathcal{T}^{i i}\left[\mathcal{G}_{e} A_{0} \mathcal{G}_{e}\right]^{i i} \mathcal{T}^{i i}\right\rangle+\sum_{k \neq i}\left\langle\mathcal{T}^{i i} \mathcal{G}_{e}^{i k} \Delta \mathcal{E}_{1}^{i k} \mathcal{G}_{e}^{k i} \mathcal{T}^{i i}\right\rangle
$$

It is worth mentioning that $\mathcal{G}_{e}$ is a diagonal block matrix while $A_{0}$ is an antidiagonal block matrix, so that $\left[\mathcal{G}_{e} A_{0} \mathcal{G}_{e}\right]^{i i}$ is a two by two antidiagonal matrix. This indicates that the solution $\Delta \mathcal{E}_{1}^{i i}$ of Eq. (44) is also an antidiagonal matrix, i.e.,

$$
\Delta \mathcal{E}_{1}^{i i}=\left(\begin{array}{cc}
0 & \Delta \mathcal{E}_{1,12}^{i i} \\
\Delta \mathcal{E}_{1,21}^{i i} & 0
\end{array}\right) .
$$

Therefore Eq. (44) is equivalent to the following two equations

$$
\begin{aligned}
\Delta \mathcal{E}_{1,12}^{i i}= & \left\langle t^{r, i i}\left[G_{e}^{r} \Gamma_{R} G_{e}^{a}\right]^{i i} t^{a, i i}\right\rangle \\
& +\sum_{k \neq i}\left\langle t^{r, i i} G_{e}^{r, i k} \Delta \mathcal{E}_{1,12}^{k k} G_{e}^{a, k i} t^{a, i i}\right\rangle
\end{aligned}
$$

$$
\begin{aligned}
\Delta \mathcal{E}_{1,21}^{i i}= & -\left\langle t^{r, i i}\left[G_{e}^{a} \Gamma_{L} G_{e}^{r}\right]^{i i} t^{a, i i}\right\rangle \\
& +\sum_{k \neq i}\left\langle t^{r, i i} G_{e}^{a, i k} \Delta \mathcal{E}_{1,21}^{k k} G_{e}^{r, k i} t^{a, i i}\right\rangle,
\end{aligned}
$$

where $t^{r, i i}$ is defined in Eq. (9) and $t^{a, i i}$ is its complex conjugate. Actually Eqs. (46) are two NVC equations to calculate vertex correction $\Omega_{N V C}$ for the disorder average of $\left\langle G^{r} \Gamma_{R} G^{a}\right\rangle$ and $\left\langle G^{a} \Gamma_{L} G^{r}\right\rangle$, respectively [27]. Note that Eqs. (46) can be solved by solving linear equations.

After solving Eqs. (41) and (46), we can now calculate $\langle\ln Z\rangle$ and hence the first order averaged cumulant. From Eq. (22), we have

$$
\begin{aligned}
\langle\ln Z\rangle & =i \lambda \operatorname{Tr}\left[\int_{0}^{1} d x\left(\mathcal{G}_{e}-\mathcal{G}_{e}\left(A_{0}-\Delta \mathcal{E}_{1}\right) \mathcal{G}_{e}\right) A_{0} x\right] \\
& =-\frac{i \lambda}{2} \operatorname{Tr}\left[\mathcal{G}_{e}\left(A_{0}-\Delta \mathcal{E}_{1}\right) \mathcal{G}_{e} A_{0}\right],
\end{aligned}
$$

where we have used the fact $\operatorname{Tr}\left[\mathcal{G}_{0} A_{0}\right]=0$. The corresponding first order cumulant is equal to

$$
\begin{aligned}
\left.\frac{\partial\langle\ln Z\rangle}{\partial i \lambda}\right|_{\lambda=0}= & \frac{1}{2} \operatorname{Tr}\left[G_{e}^{r} \Gamma_{R} G_{e}^{a} \Gamma_{L}+G_{e}^{a} \Gamma_{L} G_{e}^{r} \Gamma_{R}\right. \\
& \left.+G_{e}^{r} \Delta \mathcal{E}_{1,12} G_{e}^{a} \Gamma_{L}-G_{e}^{a} \Delta \mathcal{E}_{1,21} G_{e}^{r} \Gamma_{R}\right] .
\end{aligned}
$$

The same expression was obtained from CPA-NVC [7]. Hence we have proven that the first order cumulant calculated by FCS-CPA is equal to that of the traditional CPA-NVC method. Note that the first two terms in the right hand side of Eq. (48) describe the specular transport of electrons. While the third and fourth terms are the diffusive scattering part, these two terms describe the effect that the transverse momentum of electron is not conserved when it travels through the disordered region [28].

\section{General cumulant expansion}

In this section, we will present the general equation for calculating the renormalized on-site potential $\Delta \mathcal{E}_{j}$ up to the $j$ th order. Since the derivation is quite straightforward but tedious, we only show the final results here, and the details of derivation will be given in Appendix 1 .

Because of Eq. (28) we expand $\Delta \mathcal{E}$ in terms of $\zeta$ as $\Delta \mathcal{E}=\Delta \mathcal{E}_{0}+\Delta \mathcal{E}_{1} x \zeta+\cdots+\Delta \mathcal{E}_{j}(x \zeta)^{j}$. Substituting it into Eq. (34) and expanding further, a general equation determining $j$ th order renormalized on-site potential $\Delta \mathcal{E}_{j}^{i i}$ with $j>1$ is obtained by collecting the coefficient of $\zeta^{j}$ for different $j$ in Eq. (34) (see Appendix 1 for details)

$$
\Delta \mathcal{E}_{j}^{i i}=\sum_{k \neq i}\left\langle\mathcal{T}^{i i} \mathcal{G}_{e}^{i k} \Delta \mathcal{E}_{j}^{k k} \mathcal{G}_{e}^{k i} \mathcal{T}^{i i}\right\rangle+\mathcal{U}_{j}^{i i},
$$

and $\mathcal{U}_{j}^{i i}$ is given by

$$
\begin{aligned}
\mathcal{U}_{j}^{i i}= & -\left\langle\mathcal{T}^{i i}\left[N_{j-1} A_{0} \mathcal{G}_{e}\right]^{i i} \mathcal{T}^{i i}\right\rangle+\left\langle\mathcal{T}^{i i} \sum_{m=1}^{j-1}\left[N_{m} \Delta \mathcal{E}_{j-m} \mathcal{G}_{e}\right]^{i i} \mathcal{T}^{i i}\right\rangle \\
& -\left\langle\mathcal{T}^{i i} \sum_{m=1}^{j-1}\left[N_{m} \Delta \mathcal{E}_{j-m}\right]^{i i} Q^{i i}\right\rangle-\left\langle\mathcal{T}^{i i} \sum_{m=1}^{j-1} H_{j-m}^{i i} K_{m}^{i i}\right\rangle,
\end{aligned}
$$


where the quantity $N_{j}$ is defined as $N_{0}=\mathcal{G}_{e}, N_{1}=\mathcal{G}_{e}\left(\Delta \mathcal{E}_{1}-\right.$ $\left.A_{0}\right) \mathcal{G}_{e}$ and for $j>1$,

$$
N_{j}=\sum_{m=0}^{j-1} N_{m} \Delta \mathcal{E}_{j-m} \mathcal{G}_{e}-N_{j-1} A_{0} \mathcal{G}_{e} .
$$

The quantity $H_{j}^{i i}$ (for $j>0$ ) is defined as

$$
H_{j}^{i i}=\sum_{m=0}^{j-1}\left[N_{m}^{i i} \Delta \mathcal{E}_{j-m}^{i i}\right]-N_{j}^{i i} \Delta \overline{\mathcal{E}}_{0}^{i i},
$$

where $\Delta \overline{\mathcal{E}}_{0}^{i i}=\left(v^{i i} I-\Delta \mathcal{E}_{0}^{i i}\right)$. The quantity $K_{j}^{i i}$ (for $j>0$ ) is

$$
K_{j}^{i i}=-\mathcal{Q}^{i i} H_{j}^{i i} \mathcal{Q}^{i i}-\mathcal{Q}^{i i} \sum_{m=1}^{j-1} H_{j-m}^{i i} K_{m}^{i i} .
$$

We can also show that each matrix element of $\Delta \mathcal{E}_{j}^{i i}$ in Eq. (49) can be rewritten as a linear equation, which reads

$$
\Delta \mathcal{E}_{j, \alpha \beta}^{i i}=-\sum_{k \neq i} \mathcal{R}_{\alpha \beta}^{i k} \Delta \mathcal{E}_{j, \alpha \beta}^{k k}+\mathcal{U}_{j, \alpha \beta}^{i i},
$$

where $\alpha, \beta=1,2$ denotes the matrix elements of $\Delta \mathcal{E}_{j}^{i i}, \mathcal{R}^{i k}$, and $\mathcal{U}_{j}^{i i}$. The matrix elements $\mathcal{R}_{\alpha \beta}^{i k}$ are defined as

$$
\mathcal{R}_{\alpha \beta}^{i k}= \begin{cases}-\left(G_{e}^{r, i k}\right)^{2}\left\langle t^{r, i i} t^{r, i i}\right\rangle, & \alpha=\beta=1 \\ -\left(G_{e}^{a, i k}\right)^{2}\left\langle t^{a, i i} t^{a, i i}\right\rangle, & \alpha=\beta=2 \\ -G_{e}^{r, i k} G_{e}^{a, i k}\left\langle t^{r, i i} t^{a, i i}\right\rangle, & \text { otherwise . }\end{cases}
$$

Here we have used the property that $G_{e}^{r}\left(G_{e}^{a}\right)$ is a symmetric matrix. Furthermore, Eq. (54) can be written as several matrix equations. For example, if we consider a system with $n$ site, the equation to determine $\Delta \mathcal{E}_{j, \alpha \beta}^{i i}$ with $i=1,2, \ldots n$ can be written as

$$
\begin{aligned}
& \left(\begin{array}{c}
\Delta \mathcal{E}_{j, \alpha \beta}^{11} \\
\Delta \mathcal{E}_{j, \alpha \beta}^{22} \\
\vdots \\
\Delta \mathcal{E}_{j, \alpha \beta}^{n n}
\end{array}\right) \\
& =\left(\begin{array}{cccc}
1 & \mathcal{R}_{\alpha \beta}^{12} & \ldots & \mathcal{R}_{\alpha \beta}^{1 n} \\
\mathcal{R}_{\alpha \beta}^{21} & 1 & \ldots & \mathcal{R}_{\alpha \beta}^{2 n} \\
\vdots & \vdots & \ddots & \vdots \\
\mathcal{R}_{\alpha \beta}^{n 1} & \mathcal{R}_{\alpha \beta}^{n 2} & \cdots & 1
\end{array}\right)^{-1} \times\left(\begin{array}{c}
\mathcal{U}_{j, \alpha \beta}^{11} \\
\mathcal{U}_{j, \alpha \beta}^{22} \\
\vdots \\
\mathcal{U}_{j, \alpha \beta}^{n n}
\end{array}\right) .
\end{aligned}
$$

To summarize, the renormalized on-site potentials $\Delta \mathcal{E}_{j}$ up to $j$ th order can be easily obtained by solving the linear equation Eq. (56). To do so, we need to construct the matrix $\mathcal{R}_{\alpha \beta}$ and column vectors $\mathcal{U}_{j, \alpha \beta}$ for different $j$. Notice that $\mathcal{R}_{\alpha \beta}$ does not depend on the index $j$, which can be calculated once for all. During the calculation of $\mathcal{U}_{j, \alpha \beta}$ in Eq. (50), we have to find all the matrices $N_{m}$ in Eq. (51) and two site dependent column vectors $H_{m}$ and $K_{m}$ in Eqs. (52) and (53) for $m<j$. Here we wish to mention that the calculation of the matrix $N_{m}$ is the most time consuming part since it involves the dense matrix multiplication, while the computations of $H_{m}$ and $K_{m}$ are quite fast. The computational complexity for calculating $j$ th order cumulant amounts to $j(j+1) / 2$ matrix multiplications of order $n$.

Once we get $\Delta \mathcal{E}_{j}$, the next step is to calculate the disorder averaged CGF $\langle\ln Z\rangle$ and hence the cumulant $\left\langle C_{n}\right\rangle$ based on them. In the next subsection, a general formula to calculate both of them will be given.

\section{E. General expression for CGF}

Following the definitions in the last section, we can rewrite Eq. (22) as (see Appendix 2 for details)

$$
\langle\ln Z\rangle=\sum_{m=0}^{\infty} \frac{\left(e^{i \lambda}-1\right)^{m+1}}{2 m+2} \operatorname{Tr}\left[N_{2 m+1} A_{0}\right] .
$$

Expanding $\left(e^{i \lambda}-1\right)^{m+1}$ up to the $n$th order and using the binomial theorem, the $n$th order disorder averaged cumulant can be expressed as

$$
\left\langle C_{n}\right\rangle=\left.\frac{\partial^{n}\langle\ln Z\rangle}{(\partial i \lambda)^{n}}\right|_{\lambda=0}=\sum_{m=0}^{n-1} \frac{\beta_{m}}{2 m+2} \operatorname{Tr}\left[N_{2 m+1} A_{0}\right],
$$

where

$$
\beta_{m}=\sum_{r=0}^{m+1} C_{m+1}^{r}(m+1-r)^{n}(-1)^{r} .
$$

For instance, the first order cumulant is

$$
\left\langle C_{1}\right\rangle=\left.\frac{\partial\langle\ln Z\rangle}{\partial i \lambda}\right|_{\lambda=0}=\frac{1}{2} \operatorname{Tr}\left[N_{1} A_{0}\right],
$$

the second order cumulant (shot noise) is

$$
\left\langle C_{2}\right\rangle=\left.\frac{\partial^{2}\langle\ln Z\rangle}{(\partial i \lambda)^{2}}\right|_{\lambda=0}=\frac{1}{2} \operatorname{Tr}\left[N_{1} A_{0}+N_{3} A_{0}\right],
$$

and the third order cumulant (skewness) is

$$
\left.\frac{\partial^{3}\langle\ln Z\rangle}{(\partial i \lambda)^{3}}\right|_{\lambda=0}=\operatorname{Tr}\left[\frac{1}{2} N_{1} A_{0}+\frac{3}{2} N_{3} A_{0}+N_{5} A_{0}\right] .
$$

It is worth mentioning that in order to calculate the $n$th order averaged cumulant, we need to calculate $\Delta \mathcal{E}_{j}$ up to $j=(2 n-$ 1)th order.

\section{F. Transmission fluctuation}

In mesoscopic physics, people also use conductance (transmission) fluctuation to characterize the stochastic property of disordered systems, which is defined as $\delta T=\sqrt{\left\langle T^{2}\right\rangle-\langle T\rangle^{2}}$. Note that $\left\langle T^{2}\right\rangle$ is defined as $\left\langle[\operatorname{Tr}(\hat{T})]^{2}\right\rangle$ which is not the second order cumulant $\left\langle\operatorname{Tr}\left[\hat{T}^{2}\right]\right\rangle$ that we have discussed in the previous section. In the following, we will provide two different formulas to calculate $\left\langle T^{2}\right\rangle$ using FCS-CPA approach. The first one can be expressed using Eq. (14)

$$
\begin{aligned}
\ln Z\left(\lambda_{1}, \lambda_{2}\right)= & \sum_{n m} \ln \operatorname{Det}\left[I+\left(\begin{array}{cc}
G^{r} & 0 \\
0 & G^{a}
\end{array}\right)\right. \\
& \left.\times\left(\begin{array}{cc}
0 & \left.-X_{n m}\left(e^{i \lambda_{1}}-1\right)-X_{n m}^{\dagger}\left(e^{i \lambda_{2}}-1\right)\right) \\
\Gamma_{L} & 0
\end{array}\right)\right] \\
= & \sum_{n m} \ln \operatorname{Det}\left(I+G^{r}\left[X_{n m}\left(e^{i \lambda_{1}}-1\right)\right.\right. \\
& \left.\left.+X_{n m}^{\dagger}\left(e^{i \lambda_{2}}-1\right)\right] G^{a} \Gamma_{L}\right),
\end{aligned}
$$


where the $X_{n m}^{\dagger}$ can be calculated from $X_{n m}=\left|W_{n}\right\rangle\left\langle W_{m}\right|$ and $\Gamma_{R}=\sum_{n}\left|W_{n}\right\rangle\left\langle W_{n}\right|[7,42]$. It is easy to show that (see Appendix 3 for details)

$$
\left.\frac{\partial^{2}\langle\ln Z\rangle}{\partial i \lambda_{2} \partial i \lambda_{1}}\right|_{\lambda_{1}=0, \lambda_{2}=0}=-\left\langle T^{2}\right\rangle
$$

which is

$$
\left\langle T^{2}\right\rangle=\sum_{n m}\left\langle\operatorname{Tr}\left[G^{r} \Gamma_{L} G^{a} X_{n m} G^{r} \Gamma_{L} G^{a} X_{n m}^{\dagger}\right]\right\rangle .
$$

Note that Eq. (64) is the second order moment not the second order cumulant because of the presence of two counting fields. In this method, we have to sum up different channel numbers $n$ and $m$ and take partial derivatives with respect to $\lambda_{1}$ and $\lambda_{2}$.

In the following, we will present another generating function to calculate $\left\langle T^{2}\right\rangle$ that uses only one counting field. Note that the following identity holds,

$$
\begin{aligned}
Y X_{n m} Y= & Y X_{n m} Y\left(I+X_{n m}\right)\left(I+X_{n m}\right)^{-1} \\
= & {\left[Y\left(I+X_{n m}\right) Y\left(I+X_{n m}\right)\right.} \\
& \left.-Y I Y\left(I+X_{n m}\right)\right]\left(I+X_{n m}\right)^{-1} \\
= & \left(\mathcal{T}_{1 n m}-\mathcal{T}_{2 n m}\right)\left(I+X_{n m}\right)^{-1},
\end{aligned}
$$

where $Y=G^{r} \Gamma_{L} G^{a}, \mathcal{T}_{1 n m}=Y\left(I+X_{n m}\right) Y\left(I+X_{n m}\right)$, and $\mathcal{T}_{2 n m}=Y I Y\left(I+X_{n m}\right)$. This means that

$$
\left\langle T^{2}\right\rangle=\sum_{n m} \operatorname{Tr}\left[\left(\left\langle\mathcal{T}_{1 n m}\right\rangle-\left\langle\mathcal{T}_{2 n m}\right\rangle\right)\left(I+X_{n m}\right)^{-1} X_{n m}^{\dagger}\right] .
$$

Now we can use two generating functions to calculate the $\mathcal{T}_{1 n m}$ and $\mathcal{T}_{2 n m}$, separately. For the first term $\mathcal{T}_{1 n m}$, we use the following generating function defined as

$$
\begin{aligned}
\ln Z_{1}(\lambda) & =\ln \left[I+G\left(\begin{array}{cc}
0 & -\Gamma_{L} \\
\left(I+X_{n m}\right) & 0
\end{array}\right) \zeta\right] \\
& =\ln \left(I+G^{r} \Gamma_{L} G^{a}\left(I+X_{n m}\right)\left(e^{i \lambda}-1\right)\right) .
\end{aligned}
$$

Note that we can calculate the disorder averaged cumulant corresponding to this generating function by using the previous formalism presented in subsections $\mathrm{C}$ and D. By taking derivative with respective to $i \lambda$, it is easy to see that the disorder averaged first order cumulant is $\left\langle G^{r} \Gamma_{L} G^{a}(I+\right.$ $\left.\left.X_{n m}\right)\right\rangle$ while the second order cumulant is $\left\langle-G^{r} \Gamma_{L} G^{a}(I+\right.$ $\left.\left.X_{n m}\right) G^{r} \Gamma_{L} G^{a}\left(I+X_{n m}\right)+G^{r} \Gamma_{L} G^{a}\left(I+X_{n m}\right)\right\rangle$. So $\mathcal{T}_{1 n m}$ can be obtained by subtracting the second order cumulant from the first order cumulant.

To calculate the second term $\mathcal{T}_{2 n m}$, we introduce another generating function

$$
\begin{aligned}
\ln Z_{2}(\lambda) & =\ln \left[I+G\left(\begin{array}{cc}
0 & -\Gamma_{L} \\
I & 0
\end{array}\right) \zeta\right] \\
& =\ln \left(I+G^{r} \Gamma_{L} G^{a} I\left(e^{i \lambda}-1\right)\right) .
\end{aligned}
$$

By calculating the disorder averaged second order cumulant with respect to $\ln Z_{2}$, we can get $\left\langle G^{r} \Gamma_{L} G^{a} I G^{r} \Gamma_{L} G^{a} I\right\rangle$, from which $\mathcal{T}_{2 n m}$ can be obtained by multiplying $\left(I+X_{n m}\right)$. Finally, the transmission fluctuation can be calculated using Eq. (67).

\section{NUMERICAL RESULTS}

As an application, we have implemented the FCS-CPA formalism in the tight-binding model on the square lattice. As discussed in the section of theoretical formalism, there are two approaches to calculate the renormalized potential $\Delta \mathcal{E}$ and hence the disorder averaged cumulants using FCS-CPA. The first one is to calculate $\Delta \mathcal{E}$ by solving the CPA equation, Eq. (27), self-consistently for a given $\lambda$ and then use Eq. (30) to calculate cumulants which will be referred as FCS-CPA-I. The second one is to calculate $\Delta \mathcal{E}$ order by order in $\zeta$ from Eq. (56), which will be denoted as FCS-CPA-II.

In principle, it is straightforward to generalize our formalism within the framework of tight-binding (TB) linear muffintin orbital (LMTO) to simulate quantum transport properties of nanodevices with disorder from atomic first principles. Firstly, the charge density of disordered system can be calculated selfconsistently by using DFT within the Keldysh nonequilibrium Green's function (NEGF) formalism [28,29]. Secondly, the CPA Green's function can be calculated when self-consistency is achieved. Finally, the disorder averaged high order cumulant of conductance in nanodevices can be calculated by using the FCS-CPA approach.

In this section, we shall present our numerical results for the disorder averaged cumulants and transmission fluctuation by considering two types of disorder present in the system, i.e., Anderson and binary disorder. In the tight-binding model, the hopping energy between the nearest lattice sites is set to $t$ and the on-site energy is $4 t$.

\section{A. Anderson type of disorder}

In this subsection, we will show the numerical results for the Anderson disorder within a wide range of disorder strengths $\mathrm{W}$ (from $0.1 t$ to $5 t$ ). Note that as the disorder is turned on the clean system will be driven into the diffusive regime and finally approaches the localized regime for the large disorder strength. People usually concentrate on the quantum transport properties in the diffusive regime since universal behavior may exist in this regime. For the Anderson type of disorder, the disorder distribution function is given by

$$
\rho\left(v^{i i}\right)=\left\{\begin{array}{ll}
1 / \mathrm{W}, & -\mathrm{W} / 2 \leqslant v^{i i} \leqslant \mathrm{~W} / 2 \\
0, & \text { otherwise }
\end{array} .\right.
$$

In the numerical simulation, the incoming electron energy is set to be $E_{f}=2.97 t$ corresponding to 20 transmission channels.

As mentioned in the section of theoretical formalism, we have proposed two theoretical formalisms FCS-CPA-I and FCS-CPA-II. They should give the same results as that obtained by the traditional CPA-NVC method when calculating the disorder averaged transmission. These are useful checks to test our numerical code. In Fig. 2(a), the disorder averaged transmission versus the disorder strength $\mathrm{W}$ is presented. The following observations are in order: (1) The numerical results obtained by FCS-CPA-I, FCS-CPA-II, and traditional CPA-NVC are the same (the blue dash-dot line, orange dashed line, and yellow dot line coincide). (2) For the brute force (BF) calculation, we have calculated ten thousand configurations for the averaged transmission coefficient and a hundred thousand configurations for averaged transmission fluctuation, and the 

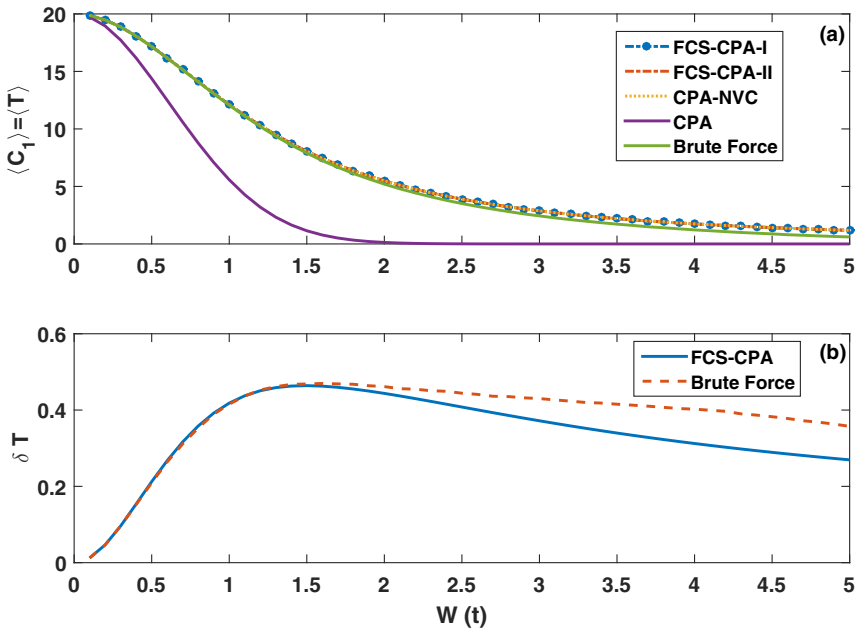

FIG. 2. Disorder averaged transmission $\langle T\rangle$ (a) and transmission fluctuation $\delta T$ (b) versus the disorder strength $\mathrm{W}$ in $30 \times 30$ square lattice system, respectively. Ten and hundred thousand configurations are calculated and averaged in the brute force calculation for (a) and (b), respectively. In (a), three curves obtained from FCS-CPA-I, FCS-CPA-II, and CAP-NVC are on top of each other.

detailed parameter settings of the brute force calculation in Fig. 2(a) have been listed in Appendix 4. Comparing with the $\mathrm{BF}$ calculation, the agreement is remarkably well for the averaged transmission coefficient. However, we do see a general trend that a larger disorder causes a larger deviation due to the nature of the CPA approximation which only considers the single site scattering event. The multiscattering is expected to play an important role when disorder strength is large. (3) In general, FCS-CPA formalism overestimates averaged transmission coefficient for large disorder strength. (4) In addition, we also plot the results of transmission by using CPA without NVC correction. This indicates that the NVC part plays an important role in calculating disorder averaged transmission [29].

Figure 3 presents the disorder averaged cumulants from second to fifth order. The numerical results obtained by FCS-CPA-I and FCS-CPA-II methods are exactly the same.
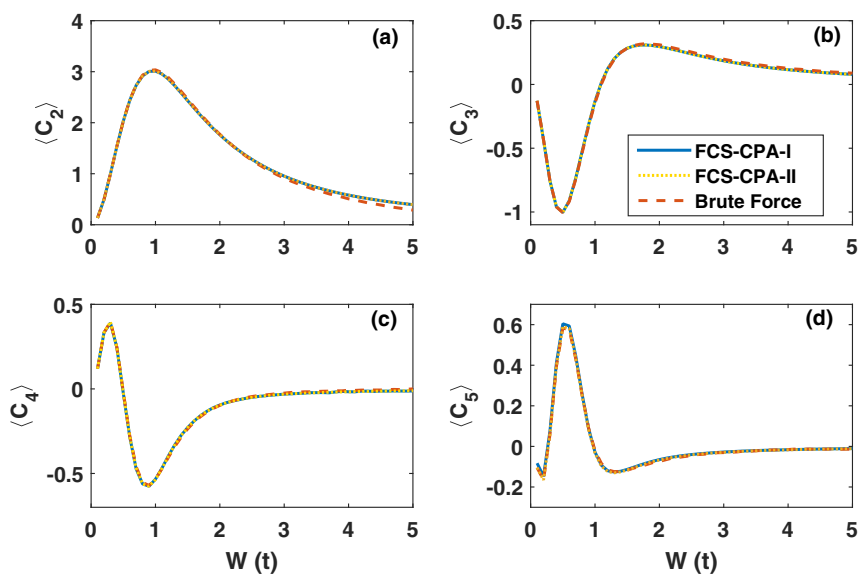

FIG. 3. Panels (a)-(d): Disorder averaged cumulants $\left\langle C_{n}\right\rangle$ when $n$ is from 2 to 5 in a $30 \times 30$ square lattice system.
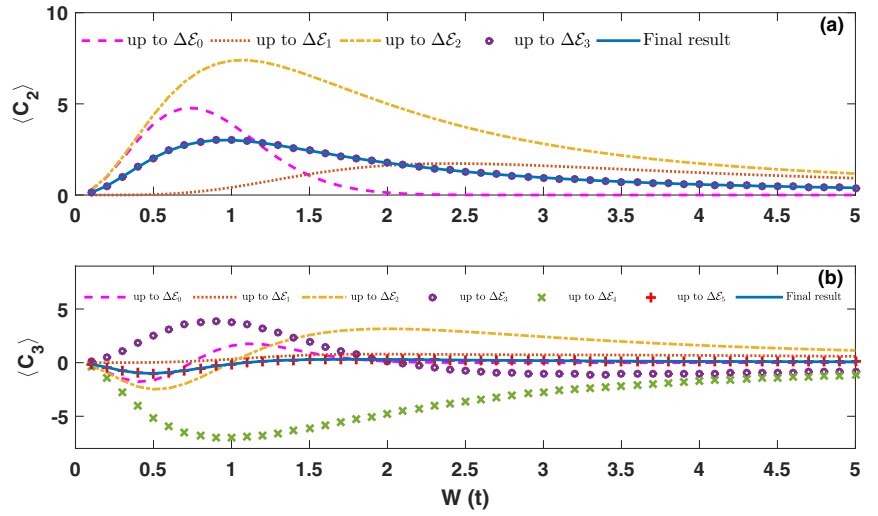

FIG. 4. Panels (a),(b): The averaged cumulants $\left\langle C_{2}\right\rangle$ and $\left\langle C_{3}\right\rangle$ up to $\Delta \mathcal{E}_{j}$ where $j$ is smaller than 6 .

Comparing the numerical results with the brute force calculation, the results from FCS-CPA methods are surprisingly accurate in the whole range of disorder strength. We see that the third to fifth order cumulant change sign when disorder strength $\mathrm{W}$ is between $0.1 t$ and $2.5 t$ while the second order cumulant is always positive. Moreover, all averaged cumulants go to zero when disorder strength becomes large enough as the system eventually enters into the localized regime.

As we have discussed in the last section, in order to calculate the $n$th order cumulant $\left\langle C_{n}\right\rangle$, one needs to calculate $\Delta \mathcal{E}_{j}$ up to $j=2 n-1$ terms in the FCS-CPA-II approach. To examine their contributions to the final disordered averaged cumulant, we take $\left\langle C_{2}\right\rangle$ and $\left\langle C_{3}\right\rangle$ as examples including contributions up to $\Delta \mathcal{E}_{j}$ for $j<6$. The numerical results are shown in Fig. 4. We see that the contributions of $\Delta \mathcal{E}_{j}$ did not decrease when $j$ is increasing. This indicates that the contributions coming from different order $\Delta \mathcal{E}_{j}$ are equally important. Therefore, one has to calculate all the required terms in order to get the correct $\left\langle C_{n}\right\rangle$. Physically, the interchannel scattering effects are included in high order $\Delta \mathcal{E}_{j}$. By including the high order contributions from $\Delta \mathcal{E}_{j}$, the disorder averaged cumulant gradually converge to the correct values. Moreover, we see that the contribution of specific $\Delta \mathcal{E}_{j}$ can be either positive or negative versus the disorder strength. For example, if we consider $\left\langle C_{2}\right\rangle$ when $\mathrm{W}$ is between $0.1 t$ and $1 t$ in Fig. 4(a), the value is decreased when the contribution $\Delta \mathcal{E}_{1}$ is considered and it will increase again when $\Delta \mathcal{E}_{2}$ is included.

As discussed above, we know that the disorder averaged high order cumulants calculated by using FCS-CPA formalism are accurate enough when the system is driven into the diffusive regime. Therefore, it would be interesting to compare the numerical results by using the FCS-CPA method with theoretical values in a quasi-one-dimensional system [43]. For this purpose, we numerically calculate the disorder averaged cumulant for the system with fixed width as 30 lattice constants and different lengths $L$. As shown in Fig. 5, we plot the normalized disorder averaged high order cumulants $\left(\left\langle C_{n}\right\rangle /\left\langle C_{1}\right\rangle, n=2,3,4,5\right)$ versus the number of disordered layers $L$. With increasing of the number of disordered layers, the normalized disorder averaged high order cumulants approach the theoretical values [43]. This also confirms the accuracy of FCS-CPA formalism. 

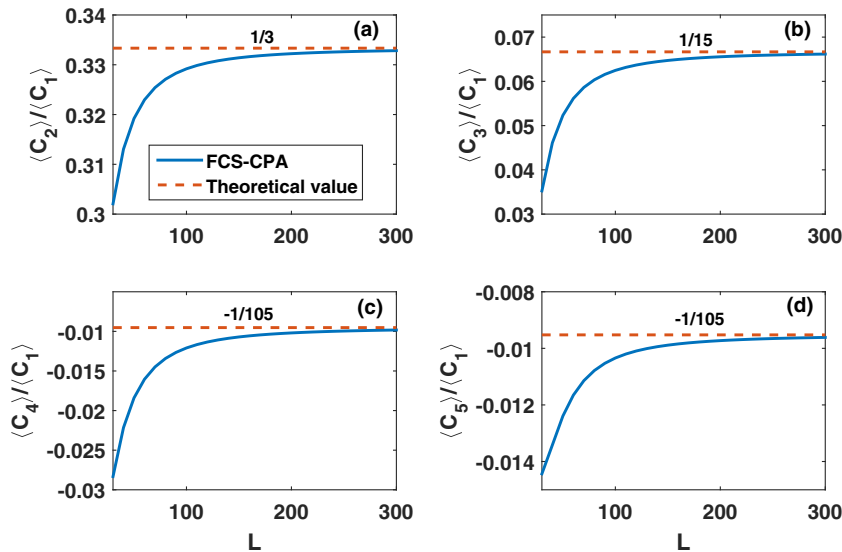

FIG. 5. Panels (a)-(d): Normalized disorder averaged high order cumulants $\left(\left\langle C_{n}\right\rangle /\left\langle C_{1}\right\rangle, n=2,3,4,5\right)$ versus the number of disordered layers $L$ in diffusive regime when $\mathrm{W}=1.5 t$. Here the width of square lattice is fixed as 30 .

Now we examine the transmission fluctuation $\delta T$ using FCS-CPA which is shown in Fig. 2(b). Comparing with BF results, the FCS-CPA can give a very accurate result when $\mathrm{W}$ is between $0.1 t$ and $1.5 t$. Further increasing disorder strength, the deviation becomes large. In addition, FCS-CPA underestimates the transmission fluctuation for large disorders. It is known that in the diffusive regime, the transmission fluctuation shows universal value (universal conductance fluctuation or UCF) [14-16]. It is known that the fluctuation plateau [near $W=1.5 t$, Fig. 2(b)] is a good estimate for UCF [19]. For a $30 \times 30$ lattice with 20 transmission channels, Fig. 2(b) estimates universal conductance (transmission) fluctuation is around 0.46 for a single spin channel which is close to the theoretical value of 0.43 for large enough transmission channels $[19,20]$. The fact that FCS-CPA agrees well with the BF fluctuation plateau region suggests that UCF can be accurately calculated using the FCS-CPA approach.

Comparing Fig. 2(b) with Fig. 3(a), we see that the performance of FCS-CPA for the shot noise is much better than that for the transmission fluctuation at large $W$. According to the definition of transmission fluctuation $\delta T=\sqrt{\left\langle T^{2}\right\rangle-\langle T\rangle^{2}}$, we have to calculate $\left\langle T^{2}\right\rangle=\left\langle[\operatorname{Tr}(\hat{T})]^{2}\right\rangle$. In eigenchannel space, the transmission matrix $\hat{T}$ is a diagonal matrix with eigenvalue $t_{n}$ which gives $\left\langle\operatorname{Tr}\left(\hat{T}^{2}\right)\right\rangle=\left\langle\sum_{n} t_{n}^{2}\right\rangle$ for the shot noise and $\left\langle T^{2}\right\rangle=\sum_{n m} t_{n} t_{m}$. The cross correlation between different transmission eigenchannels $\left\langle\sum_{n \neq m} t_{n} t_{m}\right\rangle$ is included during the calculation of $\left\langle T^{2}\right\rangle$ which is absent in shot noise calculation. This indicates that the performance of FCS-CPA on the averaged cross correlation between different transmission channels is poor. Furthermore, it is worth mentioning that the transmission distribution function $P(T)$ is difficult to be calculated by using FCS-CPA formalism, which is characterized by the transmission fluctuation, skewness, kurtosis, etc., since the cumulants defined in FCS-CPA formalism are directly expressed as the different order of transmission operator [see Eq. (18)] instead of transmission coefficient $T$. Therefore, the cumulant is in the quantum average manner. In general, it is hard to establish their direct relation just like the second cumulant $C_{2}$ and transmission fluctuation $\delta T$ discussed above.

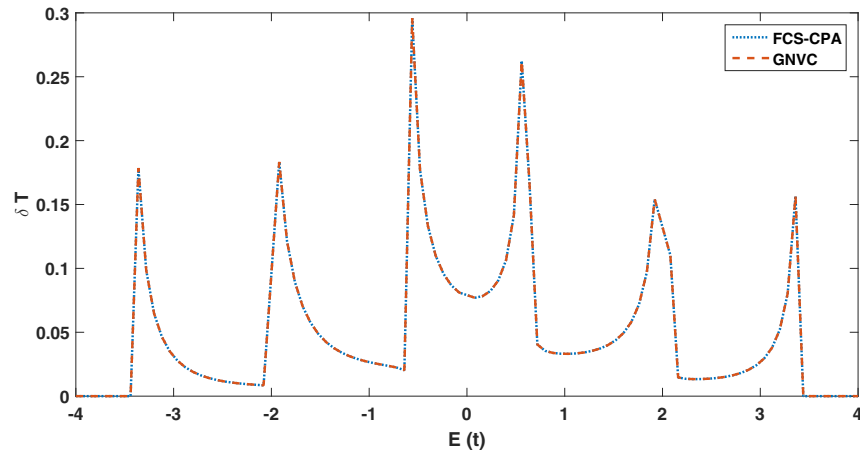

FIG. 6. The transmission fluctuations with disorder concentration $p=0.1$ in the $3 \times 3$ square lattice system when $\mathrm{W}=0.5 t$. Two curves coincide with each other.

\section{B. Binary disordered system}

In this subsection, we will present the application of the FCS-CPA formalism to calculate the binary disordered system. For the binary alloy system, the distribution function has the following form

$$
\rho\left(v^{i i}\right)=p \delta\left(v^{i i}-\mathrm{W}\right)+(1-p) \delta\left(v^{i i}-0\right),
$$

where $0 \leqslant p \leqslant 1$ is the doping concentration, and $\mathrm{W}$ is the energy difference between the dopant and the original host atom. In the numerical simulation, we consider the binary alloy system with $p=0.1,0.3,0.5$ and the energy difference $\mathrm{W}$ from $0.1 t$ to $5 t$. The incoming electron energy is set as $E_{f}=2.97 t$.

As a further test of our formalism, we compare our numerical calculation results with the previous calculation based on GNVC method in Ref. [27] where the transmission fluctuation was calculated on the $3 \times 3$ square lattice. From Fig. 6, we see clearly that our results are exactly the same as theirs.

In Fig. 7, we present FCS-CPA results comparing with the brute force results for disorder averaged cumulants up to the fourth order. We have several observations: (1) The disorder averaged cumulants obtained by FCS-CPA and the brute force
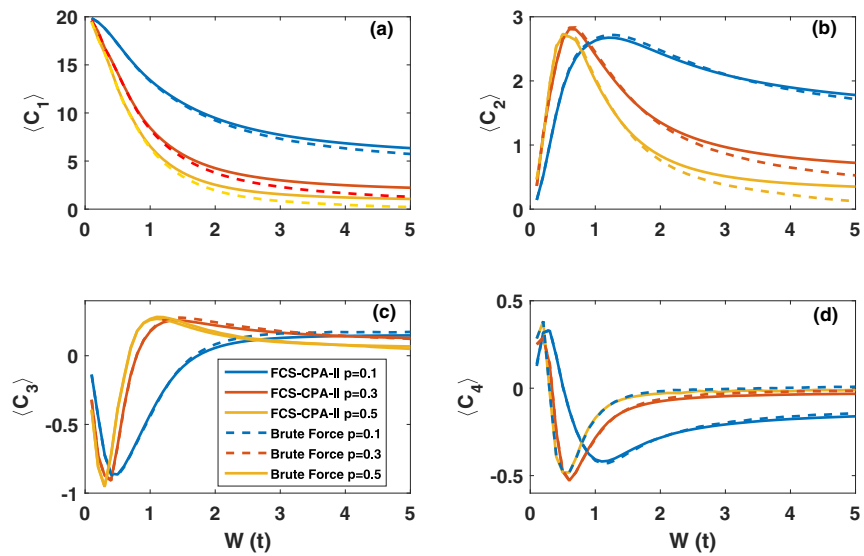

FIG. 7. Panels (a)-(d): Disorder averaged cumulants up to the fourth order in $30 \times 30$ square lattice system when the doping concentration $p=0.1,0.3,0.5$. 

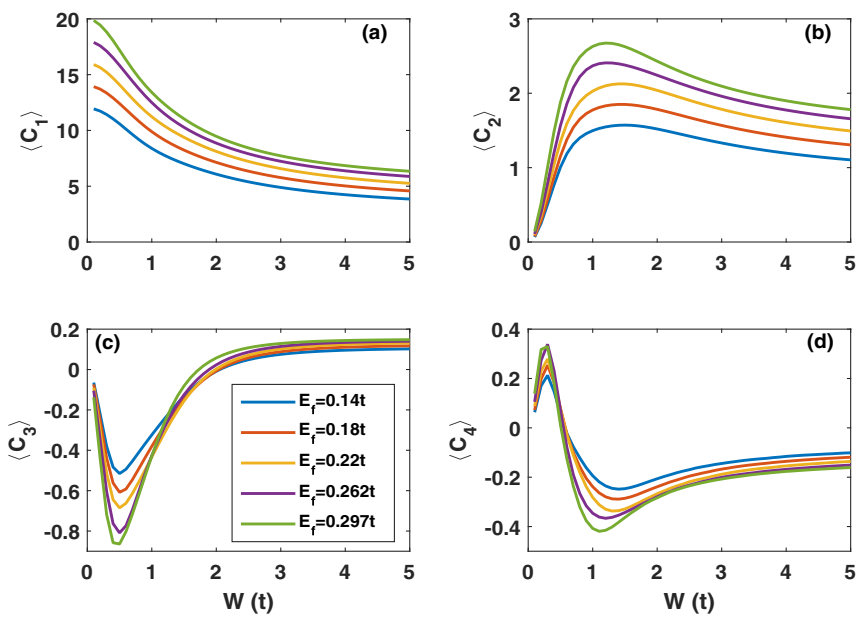

FIG. 8. Panels (a)-(d): Disorder averaged cumulants up to fourth order in $30 \times 30$ square lattice system when the electron injecting energy $E_{f}$ is changed.

method at low doping concentrations agree better than that for high doping concentrations, which is reasonable, since for the low doping concentration there are less scattering events and hence multiple scattering events are not important. (2) The first order averaged cumulant $\left\langle C_{1}\right\rangle$ decreases monotonically with energy difference $W$ while high order averaged cumulants $\left\langle C_{j}\right\rangle$ show similar behavior as that of Anderson disorders for all doping concentrations. (3) The larger the doping concentration $p$, the closer averaged cumulant $\left\langle C_{n}\right\rangle$ is to zero for large W.

Finally, we compare the averaged cumulant $\left\langle C_{n}\right\rangle$ by varying the electron incoming energy $E_{f}$. For different $E_{f}$ ranging from $1.4 t$ to $2.97 t$, the number of transmission channels changes from 12 to 20 . From Fig. 8, we see that $\left\langle C_{1}\right\rangle$ and $\left\langle C_{2}\right\rangle$ increase monotonically with respect to the increase of incoming energy. Moreover, $\left\langle C_{3}\right\rangle$ and $\left\langle C_{4}\right\rangle$ show crossover behaviors as $E$ changes when $\mathrm{W}$ is from $0.1 t$ to $2 t$.

\section{SUMMARY}

Based on the nonequilibrium Green's function, we have developed a theoretical formalism to calculate the disorder averaged cumulants of conductance using FCS framework within the coherent potential approximation. Specifically, we have mapped the problem of nonlinear functional dependence of CGF on Green's function into a linear dependent problem in high dimensional space so that the random average of CGF can be done using CPA only. We have proven analytically that for averaged transmission coefficient the FCS-CPA formalism is the same as the traditional CPA-NVC method. We have shown numerically that the conductance fluctuation obtained by the FCS-CPA method is the same as that obtained from GNVC method. In addition, the FCS-CPA formalism allows us to calculate the disorder averaged cumulants to any desired order. Furthermore we have given a general formula to calculate the renormalized potentials by solving linear equations which enables us to calculate averaged cumulants efficiently. Without loss of generality, we have implemented our theoretical formalism to the tight binding model on a square lattice to calculate the disorder averaged high order cumulants by considering two types of disorder. From numerical results, we found that our formalism can give out the very accurate results comparing with the brute force calculation.

\section{ACKNOWLEDGMENTS}

This work was financially supported by the National Natural Science Foundation of China (Grants No. 11574217 and No. 11374246), the Research Grant Council (Grant No. 17311116), the University Grant Council (Contract No. AoE/P-04/08) of the Government of HKSAR.

\section{APPENDIX}

\section{Derivation of Eq. (49)}

To obtain the explicit equation for $\Delta \mathcal{E}_{j}$, we start from expanding $\langle\mathcal{G}(x)\rangle$ in terms of $x \zeta$ in Eq. (23)

$$
\begin{aligned}
\langle\mathcal{G}(x)\rangle & =\frac{1}{\mathcal{G}_{e}^{-1}+A_{0} x \zeta-\sum_{j=1}^{\infty} \Delta \mathcal{E}_{j}(x \zeta)^{j}} \\
& =\frac{1}{I+\mathcal{G}_{e}\left(A_{0} x \zeta-\sum_{j=1}^{\infty} \Delta \mathcal{E}_{j}(x \zeta)^{j}\right)} \mathcal{G}_{e} \\
& =N_{0}+\sum_{j=1}^{\infty} N_{j}(x \zeta)^{j}
\end{aligned}
$$

where we have introduced the quantity $N_{0}=\mathcal{G}_{e}$. From Eq. (A1), we have

$I=\left[I+\sum_{j=1}^{\infty} N_{j} \mathcal{G}_{e}^{-1}(x \zeta)^{j}\right]\left[I+\mathcal{G}_{e}\left(A_{0} x \zeta-\sum_{j=1}^{\infty} \Delta \mathcal{E}_{j}(x \zeta)^{j}\right)\right]$

Further expanding Eq. (A2), we arrive at

$$
\begin{aligned}
0= & \sum_{n=1}^{\infty}\left(N_{n} \mathcal{G}_{e}^{-1}-\mathcal{G}_{e} \Delta \mathcal{E}_{n}\right)(x \zeta)^{n}+\mathcal{G}_{e} A_{0} x \zeta \\
& -\sum_{n=2}^{\infty} \sum_{j=1}^{n-1} N_{j} \Delta \mathcal{E}_{n-j}(x \zeta)^{n}+\sum_{n=2}^{\infty} N_{n-1} A_{0}(x \zeta)^{n} .
\end{aligned}
$$

Since the coefficient for each order of $\zeta$ should be zero, we obtain a recursive relation for $N_{j}$ (for $j>1$ )

$$
N_{j}=\sum_{m=0}^{j-1} N_{m} \Delta \mathcal{E}_{j-m} \mathcal{G}_{e}-N_{j-1} A_{0} \mathcal{G}_{e}
$$

and $N_{1}=\mathcal{G}_{e}\left(\Delta \mathcal{E}_{1}-A_{0}\right) \mathcal{G}_{e}$.

Furthermore, we have

$$
\langle\mathcal{G}(x)\rangle(V-\Delta \mathcal{E})=\sum_{j=0}^{\infty}\left[N_{j} \Delta \overline{\mathcal{E}}_{0}-\sum_{m=0}^{j-1} N_{m} \Delta \mathcal{E}_{j-m}\right](x \zeta)^{j}
$$

where $V$ is a diagonal matrix with $V^{i i}=v^{i i} I_{2 \times 2}$ and $\Delta \overline{\mathcal{E}}_{0}=$ $\left(V-\Delta \mathcal{E}_{0}\right)$. Then, $I-\langle\mathcal{G}(x)\rangle(V-\Delta \mathcal{E})$ in Eq. (34) can be 
expressed as

$$
I-\langle\mathcal{G}(x)\rangle(v-\Delta \mathcal{E})=\mathcal{Q}^{-1}+\sum_{j=1}^{\infty} H_{j}(x \zeta)^{j},
$$

where we have introduced $\mathcal{Q}$ and $H_{j}$, which are defined as

$$
\begin{gathered}
\mathcal{Q}^{-1}=I-N_{0} \Delta \overline{\mathcal{E}}_{0}=I-\mathcal{G}_{e}\left(V-\Delta \mathcal{E}_{0}\right), \\
H_{j}=\sum_{m=0}^{j-1}\left[N_{m} \Delta \mathcal{E}_{j-m}\right]-\left[N_{j} \Delta \overline{\mathcal{E}}_{0}\right] .
\end{gathered}
$$

For the specific lattice site $i$, we have

$$
\begin{aligned}
{\left[\frac{1}{I-\langle\mathcal{G}(x)\rangle(v-\Delta \mathcal{E})}\right]^{i i} } & =\frac{1}{\left[\mathcal{Q}^{i i}\right]^{-1}+\sum_{j=1}^{\infty} H_{j}^{i i}(x \zeta)^{j}} \\
& =\frac{1}{I+\mathcal{Q}^{i i} \sum_{j=1}^{\infty} H_{j}^{i i}(x \zeta)^{j}} \mathcal{Q}^{i i} \\
& =\mathcal{Q}^{i i}+\sum_{j=1}^{\infty} K_{j}^{i i}(x \zeta)^{j}
\end{aligned}
$$

where we defined another auxiliary quantity $K_{0}^{i i}=\mathcal{Q}^{i i}$ and $K_{j}^{i i}($ for $j>0$ )

$$
K_{j}^{i i}=-\mathcal{Q}^{i i} H_{j}^{i i} \mathcal{Q}^{i i}-\mathcal{Q}^{i i} \sum_{m=1}^{j-1} H_{j-m}^{i i} K_{m}^{i i} .
$$

Actually, disorder averaged $\left\langle K_{j}^{i i}\right\rangle$ (for $j>0$ ) is zero. This can be seen by computing the disorder average of Eq. (A9),

$$
\left\langle\left[\frac{1}{I-\langle\mathcal{G}(x)\rangle(V-\Delta \mathcal{E})}\right]^{i i}\right\rangle=\left\langle\mathcal{Q}^{i i}\right\rangle+\sum_{j=1}^{\infty}\left\langle K_{j}^{i i}\right\rangle(x \zeta)^{j} .
$$

Here we will show the left hand side of Eq. (A11) is identity. From Eq. (27), we have

$$
\begin{aligned}
0= & \left\langle\left(v^{i i} I-\Delta \mathcal{E}^{i i}\right)\left[I-\langle\mathcal{G}(x)\rangle^{i i}\left(v^{i i} I-\Delta \mathcal{E}^{i i}\right)\right]^{-1}\right\rangle \\
= & \left\langle\left(v^{i i} I-\Delta \mathcal{E}^{i i}\right)\left[\left(\langle\mathcal{G}(x)\rangle^{i i}\right)^{-1}-\left(v^{i i}-\Delta \mathcal{E}^{i i}\right)\right]^{-1}\right. \\
& \left.\times\left(\langle\mathcal{G}(x)\rangle^{i i}\right)^{-1}\right\rangle \\
= & \left\langle-I+\left[\frac{1}{I-\langle\mathcal{G}(x)\rangle(V-\Delta \mathcal{E})}\right]^{i i}\right\rangle\left(\langle\mathcal{G}(x)\rangle^{i i}\right)^{-1} .
\end{aligned}
$$

Therefore the left hand side of Eq. (A11) is identity and similarly we can prove $\left\langle\mathcal{Q}^{i i}\right\rangle=I$. Therefore, the second term of the right side $\left\langle K_{j}^{i i}\right\rangle(j>0)$ should be zero.

Using Eq. (A5) and auxiliary quantities $\mathcal{Q}^{i i}$ and $K_{j}^{i i}$, we rewrite Eq. (34) as

$$
\begin{aligned}
0= & \left\langle\left(\Delta \overline{\mathcal{E}}_{0}^{i i}-\sum_{j=1}^{\infty} \Delta \mathcal{E}_{j}^{i i}(x \zeta)^{j}\right)\left(\mathcal{Q}^{i i}+\sum_{j=1}^{\infty} K_{j}^{i i}(x \zeta)^{j}\right)\right\rangle \\
= & \left\langle\Delta \overline{\mathcal{E}}_{0}^{i i} \mathcal{Q}^{i i}\right\rangle-\left\langle\Delta \mathcal{E}_{1}^{i i} \mathcal{Q}^{i i}-\Delta \overline{\mathcal{E}}_{0}^{i i} K_{1}^{i i}\right\rangle x \zeta \\
& -\sum_{j=2}^{\infty}\left(\sum_{m=1}^{j} \Delta \mathcal{E}_{m}^{i i} K_{j-m}^{i i}-\Delta \overline{\mathcal{E}}_{0}^{i i} K_{j}^{i i}\right)(x \zeta)^{j} .
\end{aligned}
$$

Note that the coefficient of each order of $(x \zeta)^{j}$ in Eq. (A13) is equal to zero. The first and second terms in the right hand side of Eq. (A13) lead to the Eq. (39) for $\mathcal{E}_{0}^{i i}$ and Eq. (44) for $\mathcal{E}_{1}^{i i}$, respectively. In general, equation of $\mathcal{E}_{j}^{i i}$ (for $j>1$ ) can be expressed as

$$
-\Delta \mathcal{E}_{j}^{i i}\left\langle K_{0}^{i i}\right\rangle-\sum_{m=1}^{j-1} \Delta \mathcal{E}_{m}^{i i}\left\langle K_{j-m}^{i i}\right\rangle+\left\langle\Delta \overline{\mathcal{E}}_{0}^{i i} K_{j}^{i i}\right\rangle=0 .
$$

Using the fact that $\left\langle K_{0}^{i i}\right\rangle=I$ and $\left\langle K_{j}^{i i}\right\rangle=0(j>0)$ and plugging Eq. (A10) into Eq. (A14), we finally arrive at

$$
\Delta \mathcal{E}_{j}^{i i}=\sum_{k \neq i}\left\langle\mathcal{T}^{i i} \mathcal{G}_{e}^{i k} \Delta \mathcal{E}_{j}^{k k} \mathcal{G}_{e}^{k i} \mathcal{T}^{i i}\right\rangle+\mathcal{U}_{j}^{i i},
$$

where $\mathcal{U}_{j}^{i i}$ is

$$
\begin{aligned}
\mathcal{U}_{j}^{i i}= & -\left\langle\mathcal{T}^{i i}\left[N_{j-1} A_{0} \mathcal{G}_{e}\right]^{i i} \mathcal{T}^{i i}\right\rangle+\left\langle\mathcal{T}^{i i} \sum_{m=1}^{j-1}\left[N_{m} \Delta \mathcal{E}_{j-m} \mathcal{G}_{e}\right]^{i i} \mathcal{T}^{i i}\right\rangle \\
& -\left\langle\mathcal{T}^{i i} \sum_{m=1}^{j-1}\left[N_{m} \Delta \mathcal{E}_{j-m}\right]^{i i} Q^{i i}\right\rangle-\left\langle\mathcal{T}^{i i} \sum_{m=1}^{j-1} H_{j-m}^{i i} K_{m}^{i i}\right\rangle .
\end{aligned}
$$

\section{Derivation of Eq. (57)}

From Eq. (22), we have

$$
\begin{aligned}
\langle\ln Z\rangle & =\operatorname{Tr}\left[\int_{0}^{1} d x\left(N_{0}+\sum_{j=1}^{\infty} N_{j} x^{j} \zeta^{j}\right) A_{0} \zeta\right] \\
& =\operatorname{Tr}\left[\sum_{n=0}^{\infty} \int_{0}^{1} d x N_{2 n+1}\left(e^{i \lambda}-1\right)^{n+1} x^{2 n+1} A_{0}\right] \\
& =\sum_{n=0}^{\infty} \frac{\left(e^{i \lambda}-1\right)^{n+1}}{2 n+2} \operatorname{Tr}\left[N_{2 n+1} A_{0}\right],
\end{aligned}
$$

where we have used the property that the $N_{2 n}$ is a block diagonal matrix; $A_{0}$ is a block antidiagonal matrix and hence $\operatorname{Tr}\left[N_{2 n} A_{0}\right]=0$.

\section{Derivation of Eq. (64)}

Taking the derivative of generating function $\ln Z$ in Eq. (63) with respective to $i \lambda_{1}$, we have

$$
\begin{aligned}
& \left.\frac{\partial \ln Z}{\partial i \lambda_{1}}\right|_{\lambda_{1}=0} \\
& =\sum_{n m} \frac{\partial}{\partial i \lambda_{1}}\left[\operatorname { l n } \operatorname { D e t } \left(I+G^{r}\left[X_{n m}\left(e^{i \lambda_{1}}-1\right)\right.\right.\right. \\
& \left.\left.\left.\quad+X_{n m}^{\dagger}\left(e^{i \lambda_{2}}-1\right)\right] G^{a} \Gamma_{L}\right)\right]\left.\right|_{\lambda_{1}=0} \\
& =\sum_{n m} \operatorname{Tr}\left(\frac{1}{I+G^{r} X_{n m}^{\dagger}\left(e^{i \lambda_{2}}-1\right) G^{a} \Gamma_{L}} G^{r} X_{n m} G^{a} \Gamma_{L}\right) .
\end{aligned}
$$


TABLE I. Parameter settings of the brute force calculation in Fig. 2(a).

\begin{tabular}{lc}
\hline \hline & Value \\
\hline Lattice of central region & $30 \times 30$ \\
Disorder strength & $0.1 t$ to $5 t$ \\
Incoming electron energy & $E_{f}=2.97 t$ \\
Configuration number & 10000 \\
\hline \hline
\end{tabular}

We then take the derivative of Eq. (A18) with respect to $i \lambda_{2}$

$$
\begin{aligned}
& \left.\frac{\partial}{\partial i \lambda_{2}}\left(\left.\frac{\partial \ln Z}{\partial i \lambda_{1}}\right|_{\lambda_{1}=0}\right)\right|_{\lambda_{2}=0} \\
& =-\sum_{n m} \operatorname{Tr}\left(G^{r} X_{n m} G^{a} \Gamma_{L} G^{r} X_{n m}^{\dagger} G^{a} \Gamma_{L}\right)
\end{aligned}
$$

$$
\begin{aligned}
& =-\sum_{m}\left\langle W_{m}\left|G^{a} \Gamma_{L} G^{r}\right| W_{m}\right\rangle \sum_{n}\left\langle W_{n}\left|G^{a} \Gamma_{L} G^{r}\right| W_{n}\right\rangle \\
& =-\operatorname{Tr}\left[G^{a} \Gamma_{L} G^{r} \Gamma_{R}\right] \times \operatorname{Tr}\left[G^{a} \Gamma_{L} G^{r} \Gamma_{R}\right],
\end{aligned}
$$

where we have used the fact that $X_{n m}=\left|W_{n}\right\rangle\left\langle W_{m}\right|$ and $\Gamma_{R}=\sum_{n}\left|W_{n}\right\rangle\left\langle W_{n}\right|$. Taking disorder average on both sides of Eq. (A19), we can finally arrive at Eq. (64).

\section{Summary of parameter settings in brute force calculations}

In this Appendix, we list the parameter settings in the brute force calculation of Fig. 2(a) (Table I). For other figures, we also use the same parameters if not specified.
[1] A. M. Bratkovsky, Phys. Rev. B 56, 2344 (1997).

[2] H. Ohno, Science 281, 951 (1998).

[3] T. Markussen, R. Rurali, A.-P. Jauho, and M. Brandbyge, Phys. Rev. Lett. 99, 076803 (2007).

[4] F. M. Xu and J. Wang, Phys. Rev. B 89, 245430 (2014).

[5] A. Asenov, IEEE Trans. Electron Devices 45, 2505 (1998).

[6] M. Poljak, M. Wang, E. B. Song, T. Suligoj, and K. Wang, Solid-State Electron. 84, 103 (2013).

[7] Y. Zhu, L. Liu, and H. Guo, Phys. Rev. B 88, 085420 (2013).

[8] J. N. Zhuang, Y. Wang, Y. Zhou, J. Wang, and H. Guo, Front. Phys. 12, 127304 (2017).

[9] P. W. Anderson, Phys. Rev. 109, 1492 (1958).

[10] P. W. Anderson, Phys. Rev. 124, 41 (1961).

[11] Y. X. Xing, L. Zhang, and J. Wang, Phys. Rev. B 84, 035110 (2011).

[12] J. Li, R.-L. Chu, J. K. Jain, and S.-Q. Shen, Phys. Rev. Lett. 102, 136806 (2009).

[13] L. Zhang, J. N. Zhuang, Y. X. Xing, J. Li, J. Wang, and H. Guo, Phys. Rev. B 89, 245107 (2014).

[14] B. L. Altshuler, JETP Lett. 41, 648 (1985).

[15] P. A. Lee and A. D. Stone, Phys. Rev. Lett. 55, 1622 (1985).

[16] P. A. Lee, A. D. Stone, and H. Fukuyama, Phys. Rev. B 35, 1039 (1987).

[17] W. Ren, Z. H. Qiao, J. Wang, Q. F. Sun, and H. Guo, Phys. Rev. Lett. 97, 066603 (2006).

[18] Z. H. Qiao, J. Wang, Y. D. Wei, and H. Guo, Phys. Rev. Lett. 101, 016804 (2008)

[19] Z. H. Qiao, Y. X. Xing, and J. Wang, Phys. Rev. B 81, 085114 (2010).

[20] C. W. J. Beenakker, Rev. Mod. Phys. 69, 731 (1997).

[21] N. G. van Kampen, Stochastic Processes in Physics and Chemistry (Elsevier, Amsterdam, 2007).

[22] Ya. Blanter and M. Büttiker, Phys. Rep. 336, 1 (2000).

[23] P. Soven, Phys. Rev. 156, 809 (1967).
[24] D. W. Taylor, Phys. Rev. 156, 1017 (1967).

[25] B. Velicky, Phys. Rev. 184, 614 (1969).

[26] R. J. Elliott, J. A. Krumhansl, and P. L. Leath, Rev. Mod. Phys. 46, 465 (1974).

[27] J. Yan and Y. Q. Ke, Phys. Rev. B 94, 045424 (2016).

[28] Y. Ke, K. Xia, and H. Guo, Phys. Rev. Lett. 100, 166805 (2008).

[29] Y. Ke, K. Xia, and H. Guo, Phys. Rev. Lett. 105, 236801 (2010).

[30] A. V. Kalitsov, M. G. Chshiev, and J. P. Velev, Phys. Rev. B 85, 235111 (2012).

[31] Y. Zhu, L. Liu, and H. Guo, Phys. Rev. B 88, 205415 (2013).

[32] C. Zhou, X. Chen, and H. Guo, Phys. Rev. B 94, 075426 (2016).

[33] J. Yan, S. Wang, K. Xia, and Y. Ke, Phys. Rev. B 95, 125428 (2017).

[34] C. Zhou and H. Guo, Phys. Rev. B 95, 035126 (2017).

[35] L. S. Levitov, in Quantum Noise in Mesoscopic Physics, edited by Yu. V. Nazarov, NATO Science Series II, Vol. 97 (Kluwer, Dordrecht, 2003).

[36] L. S. Levitov and G. B. Lesovik, Pis'ma Zh. Eksp. Teor. Fiz. 58, 225 (1993) [JETP Lett. 58, 230 (1993)].

[37] L. S. Levitov, H.-W. Lee, and G. B. Lesovik, J. Math. Phys. 37, 4845 (1996).

[38] J. N. Zhuang and J. Wang, J. Appl. Phys. 114, 063708 (2013).

[39] The CPA can be generalized to include the nonlocal correlations that involves different scatterers, and the renormalized quantity $\Delta E$ have off-diagonal elements [44].

[40] P. Sheng, Introduction to Wave Scattering, Localization and Mesoscopic Phenomena (Springer, New York, 2006).

[41] G. M. Tang and J. Wang, Phys. Rev. B 90, 195422 (2014).

[42] J. Wang and H. Guo, Phys. Rev. B 79, 045119 (2009).

[43] H. Lee, L. S. Levitov, and A. Y. Yakovets, Phys. Rev. B 51, 4079 (1995).

[44] M. Jarrell and H. R. Krishnamurthy, Phys. Rev. B 63, 125102 (2001). 